\title{
Preparation and application of a "clickable" acceptor for enzymatic synthesis of heparin oligosaccharides
}

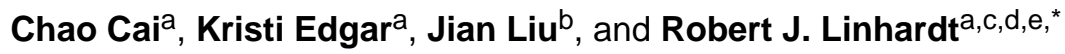 \\ aDepartment of Chemistry and Chemical Biology, Center for Biotechnology and Interdisciplinary \\ Studies, Rensselaer Polytechnic Institute, Troy, NY 12180, USA \\ ${ }^{b}$ Division of Chemical Biology and Medicinal Chemistry, Edelman School of Pharmacy, University \\ of North Carolina, Chapel Hill, NC 27599, USA \\ 'Department of Biology, Center for Biotechnology and Interdisciplinary Studies, Rensselaer \\ Polytechnic Institute, Troy, NY 12180, USA \\ ${ }^{\mathrm{d} D e p a r t m e n t}$ of Chemical and Biological Engineering, Center for Biotechnology and \\ Interdisciplinary Studies, Rensselaer Polytechnic Institute, Troy, NY 12180, USA \\ eDepartment of Biomedical Engineering, Center for Biotechnology and Interdisciplinary Studies, \\ Rensselaer Polytechnic Institute, Troy, NY 12180, USA
}

\begin{abstract}
A "clickable" disaccharide was prepared by treating the aldehyde precursor with hydroxylamine, followed by the catalytic hydrogenation and diazotransfer reaction. This disaccharide was successfully applied to the elongation of the backbone construction of ultralow molecular weight (ULMW) heparins using two bacterial glycosyl transferases, $N$-acetyl glucosaminyl transferase from Escherichia coli K5 (KfiA) and heparosan synthase-2 (pmHS2) from Pasteurella multocida.
\end{abstract}

\section{Keywords}

Heparan sulfate; Heparin; Heparosan; Depolymerization; Azido-clickable acceptor; Enzymatic glycosylation

\section{Introduction}

Heparan sulfate (HS) and heparin are linear, highly sulfated, anionic polysaccharides that belong to the glycosaminoglycan (GAG) family. ${ }^{1}$ These GAGs are composed of a repeating disaccharide motif of a glucuronic acid (GlcA) or an iduronic acid (IdoA) residue and a glucosamine residue, including $N$-acetylglucosamine (GlcNAc), $N$-sulfoglucosamine (GlcNS) or $N$-unsubstituted glucosamine $\left(\mathrm{GlcNH}_{2}\right){ }^{2} \mathrm{HS}$ is an abundant GAG on the surface of mammalian cells and in the extracellular matrix. It plays regulatory roles in several

\footnotetext{
(C) 2013 Elsevier Ltd. All rights reserved.

Corresponding author: Tel.: 518-276-3404; Fax: 518-276-3405. linhar@ rpi.edu.

Supplementary data

Supplementary data associated with this article can be found, in the online version, at

Publisher's Disclaimer: This is a PDF file of an unedited manuscript that has been accepted for publication. As a service to our customers we are providing this early version of the manuscript. The manuscript will undergo copyediting, typesetting, and review of the resulting proof before it is published in its final citable form. Please note that during the production process errors may be discovered which could affect the content, and all legal disclaimers that apply to the journal pertain.
} 
pathophysiological processes, such as development, angiogenesis, blood coagulation, and tumor metastasis. $3,4,5$

Preparation of pure GAG oligosaccharides is a major challenge for carbohydrate chemists $^{6,7,8}$ because traditional chemical synthesis of structurally defined GAGs relies on the efficient introduction of protecting groups, epimerization of GlcA to IdoA, stereoselective glycosylation, the efficient removal of protecting groups and sulfonation, requiring a large number of synthetic steps to furnish the product. ${ }^{9,10,11}$ The biosynthesis of GAGs in the Golgi of eukaryotic cells suggests a more efficient enzymatic route, which includes the building of the polysaccharide backbone, as well as introducing sulfo groups and IdoA residues. ${ }^{12,13}$ Employing a chemoenzymatic approach in our previous work, we successfully synthesized the ULMW heparin heptasaccharides with 10 and 12 steps in $45 \%$ and $37 \%$ overall yields based on the disaccharide acceptor degraded from heparosan (Figure 1), which showed very similar binding affinities and in vitro anti-Xa activities comparing with the commercial drug Arixtra. ${ }^{14,15}$

The chemoenzymatic synthesis of structurally defined heparin oligosaccharide glycoconjugates or immobilized heparin oligosaccharides might be accomplished through the introduction of a reactive site into the heparin oligosaccharide. A heparin oligosaccharide backbone can be elongated on a disaccharide acceptor to achieve this goal. ${ }^{16}$ We report the synthesis of a disaccharide acceptor with an azido group as a reactive site at the reducing end (Figure 1). This disaccharide acceptor can be applied to a "click" reaction or released as a free amine group for diverse conjugation. This modified disaccharide is quite suitable for use as an extendable acceptor for the backbone elongation for the construction of ultralow molecular weight (ULMW) heparins.

\section{Results and discussion}

Heparosan $(\rightarrow 4)$ GlcA $(1 \rightarrow 4)$ GlcNAc $(1 \rightarrow)_{\mathrm{n}}{ }^{17}$ has been prepared from the Escherichia coli $\mathrm{K} 5$ strain $^{18}$ and isolated at kilogram scale and was employed as starting material for our disaccharide acceptor target (Scheme 1). Heparosan was dissolved in $2 \mathrm{M} \mathrm{NaOH}$ solution under $60^{\circ} \mathrm{C}$ for $24 \mathrm{~h}$ to obtain the $N$-deacetylated heparosan polysaccharide according previously described methods. ${ }^{19,20}$ The $N$-deacetylated heparosan polysaccharide was deaminated with nitrous acid at low $\mathrm{pH}(\sim 4.5)$, and neutralized with $2 \mathrm{M} \mathrm{NaOH}$ to furnish disaccharide $4($ GlcA $(1 \rightarrow 4)$ anhydromannose $($ AnMan $)){ }^{21,22}$ We found that the free aldehyde group at the reducing end of disaccharide $\mathbf{4}$ was primarily obtained as the hydrate form, hemiacetal 5 . Hemiacetal 5 was identified by its ${ }^{1} \mathrm{H}$ NMR and mass spectrum, which showed the disappearance of the characteristic aldehyde peak, at $\sim 9 \mathrm{ppm}$, and displayed the hydrate form $\left[\mathrm{M}+\mathrm{H}_{2} \mathrm{O}\right]^{-}$. The $\beta$-configuration of the glycosidic linkage was confirmed on the basis of the $7.8 \mathrm{~Hz} J_{1,2}$ coupling constant, which indicates that the $\mathrm{H}-1^{\mathrm{II}}$ and $\mathrm{H}-2^{\mathrm{II}}$ atoms are in a trans relationship to one another. ${ }^{23}$ Reductive amination of $\mathbf{5}$ was initially attempted with sodium cyanoborohydride, however, low yields were achieved using both aliphatic and aromatic amines. Treatment of $\mathbf{5}$ with hydroxylamine in aqueous sodium acetate solution gave significantly improved yields ( $90 \%)$, resulting in the formation of oxime 6 as a mixture of the $E$ and $Z$ isomers. ${ }^{24}$ After the size exclusion chromatography, the ${ }^{1} \mathrm{H} \mathrm{NMR}$ spectrum of oxime 6 showed doublets at $7.44(J=3.78 \mathrm{~Hz})$ and $6.84(J=6.88 \mathrm{~Hz})$ corresponding to the presence of the $\mathrm{C} H=\mathrm{N}$ group in an $(E)$-oxime to $(Z)$-oxime ratio of 7:2.

Reduction of oxime 6 was carried out smoothly employing hydrogen with $\mathrm{Pd}(\mathrm{OH})_{2} / \mathrm{C}$ catalyst affording the corresponding amino derivative 7 in $91 \%$ yield. The structure of 7 was confirmed by ${ }^{1} \mathrm{H}$ and 2D NMR spectroscopy as it showed the disappearance of the $\mathrm{CH}=\mathrm{N}$ proton and the appearance of a multiplet at $\sim 3.6 \mathrm{ppm}$, attributable to a newly formed $\mathrm{CH}_{2}$ group. Conversion of the amino group to an azido group was subsequently accomplished by 
treating 7 with sodium azide, $\mathrm{Tf}_{2} \mathrm{O}$ and catalytic $\mathrm{CuSO}_{4}$ in a combined $\mathrm{CH}_{2} \mathrm{Cl}_{2} / \mathrm{MeOH} / \mathrm{H}_{2} \mathrm{O}$ solution, furnishing compound $\mathbf{1}$ in $88 \%$ yield. ${ }^{25}$ The ${ }^{13} \mathrm{C}$ NMR spectrum of compound $\mathbf{1}$ showed that $\mathrm{C}-1^{\mathrm{I}}$ shifted to $51.4 \mathrm{ppm}$, corresponding to a $\mathrm{CH}_{2}$ group after introducing an azido group at the reducing end. Compound $\mathbf{1}$, an azido-functional acceptor, could be employed to construct heparin oligosaccharide backbones. However, this disaccharide acceptor always contained some impurities that were difficult to remove by the size exclusion chromatography when performing large-scale reactions. Consequently, we protected the carboxyl group with a methyl ester in $95 \%$ yield under acidic conditions and the free hydroxyl groups as acetyl esters in quantitative yield to obtain compound $\mathbf{8}$, which was easily purified on a silica column. Compound $\mathbf{8}$ was then quantitatively deprotected by treating with $2 \mathrm{M} \mathrm{NaOH}$ and desalted to obtain pure disaccharide acceptor $\mathbf{1}$ (Scheme 2).

Next, the heparin oligosaccharide backbone was prepared by enzymatic glycosylation of disaccharide acceptor $\mathbf{1}$. Disaccharide 1 (GlcA-AnMan- $\mathrm{N}_{3}$ ) was incubated overnight with UDP-GlcNTFA and KfiA at room temperature to furnish the trisaccharide product. Reversephase ion-pairing HPLC (RPIP-HPLC) was employed to monitor the generation of uridine diphosphate (UDP). Here, we employed an unnatural UDP-GlcNTFA donor, as it can be readily converted to an $\mathrm{N}$-sulfoglucosamine residue in a subsequent step. ${ }^{14}$ Afterwards, one equivalent of UDP-GlcA was added into the above mixture and incubated for $4-5 \mathrm{~h}$ in the presence of pmHS2, followed by addition of a second equivalent of UDP-GlcUA and additional pmHS2 to ensure reaction completion. The resulting tetrasaccharide 9 was recovered by size exclusion chromatography. The structures of the trisaccharide intermediate and tetrasaccharide 9 were both confirmed by HR ESI-MS and NMR spectroscopy.

\section{Conclusions}

We have developed a practical route including acidic depolymerization, oximation, hydrogenation and diazotransfer reaction, towards the large scale preparation of the $\mathrm{N}_{3^{-}}$ containing "clickable" acceptor, which was also successfully applied on the backbone construction of ultralow molecular weight (ULMW) heparin oligosaccharide employing the enzymatic glycosylation. Further elongation and sulfonation of this tetrasaccharide acceptor is ongoing.

\section{Experimental}

\subsection{General Methods}

${ }^{1} \mathrm{H}$ and ${ }^{13} \mathrm{C}$ NMR spectra were recorded at $600 \mathrm{MHz}$ for ${ }^{1} \mathrm{H}$ NMR, $150 \mathrm{MHz}$ for ${ }^{13} \mathrm{C}$ NMR or $800 \mathrm{MHz}$ for ${ }^{1} \mathrm{H}$ NMR, $200 \mathrm{MHz}$ for ${ }^{13} \mathrm{C}$ NMR with Topsin 2.1 software. Mass data were acquired by high-resolution ESI-MS. Thin-layer chromatography (TLC) was carried out using plates of silica gel 60 with fluorescent indicator and revealed with UV light (254 nm) when possible and Von's reagent or ninhydrin solution in ethanol. Flash chromatography was performed using silica gel 230-400 mesh. Yields are given after purification, unless otherwise noted. When reactions were performed under anhydrous conditions, the mixtures were maintained under argon.

\section{2. $\mathrm{N}$-Deacetylation of Heparosan (3)}

Heparosan $2(3.3 \mathrm{~g}, 0.22 \mathrm{mmol})$ dissolved in $2 \mathrm{M} \mathrm{NaOH}(165 \mathrm{~mL})$ was heated at $60{ }^{\circ} \mathrm{C}$ until the solution is clear. After stirring for another $24 \mathrm{~h}$ under an argon atmosphere at $60{ }^{\circ} \mathrm{C}$, the solution was dialyzed with 1000 molecular weight cutoff (MWCO) dialysis membrane against double-distilled water for $24 \mathrm{~h}$. Then, the dialysate was lyophilized to give the $N$ - 
deactylated heparosan (3) as yellow powder. Spectra were in agreement with reported data. $^{20}$

\section{3. $\beta$-D-glucopyranosiduronate-( $\rightarrow 4$ )-2,5-Anhydro-D-mannose (4)}

A $50 \mathrm{~mL}$ solution of 2:5 $\left(1 \mathrm{M} \mathrm{H}_{2} \mathrm{SO}_{4}: 5.5 \mathrm{M} \mathrm{NaNO}_{2}\right)$ was added to dry $\mathrm{N}$-deacetylated heparosan 3 ( $2.0 \mathrm{~g}, 0.13 \mathrm{mmol})$, and vigorously stirred under ice bath for $30 \mathrm{~min}$. After the $\mathrm{pH}$ was adjusted to neutral with $30 \mathrm{~mL}$ of 3:5:5 $\left(1 \mathrm{M} \mathrm{Na}_{2} \mathrm{CO}_{3}\right.$ : dd $\left.\mathrm{H}_{2} \mathrm{O}: 1 \mathrm{M} \mathrm{NaHCO}_{3}\right)$, the solution was dialyzed using 1000 molecular weight cutoff (MWCO) dialysis membrane against double-distilled water for $4 \mathrm{~h}$ and the dialysate was lyophilized to give the crude product 4 as off-white powder $\left(\mathrm{BuOH} / \mathrm{HCOOH} / \mathrm{H}_{2} \mathrm{O}=4: 8: 1, \mathrm{R}_{\mathrm{f}}=0.44\right) .{ }^{1} \mathrm{H}$ NMR (600 $\left.\mathrm{MHz}, \mathrm{D}_{2} \mathrm{O}\right): \delta 4.95\left(\mathrm{~d}, 1 \mathrm{H}, J=5.5 \mathrm{~Hz}, \mathrm{H}-2^{\mathrm{I}}\right), 4.36\left(\mathrm{~d}, 1 \mathrm{H}, \mathrm{J}=7.8 \mathrm{~Hz}, \mathrm{H}-1^{\mathrm{II}}\right), 4.23(\mathrm{t}, 1 \mathrm{H}, J$ $\left.=5.0 \mathrm{~Hz}, \mathrm{H}-3^{\mathrm{I}}\right), 3.99\left(\mathrm{~m}, 1 \mathrm{H}, \mathrm{H}-5^{\mathrm{I}}\right), 3.97\left(\mathrm{t}, 1 \mathrm{H}, J=5.6 \mathrm{~Hz}, \mathrm{H}-4^{\mathrm{I}}\right), 3.58-3.64(\mathrm{~m}, 3 \mathrm{H}$, H-5 $\left.{ }^{\mathrm{II}}, \mathrm{H}-6 \mathrm{~b}^{\mathrm{I}}, \mathrm{H}-6 \mathrm{a}^{\mathrm{I}}\right), 3.36-3.40\left(\mathrm{~m}, 2 \mathrm{H}, \mathrm{H}-3^{\mathrm{II}}\right), 3.20\left(\mathrm{t}, 1 \mathrm{H}, J=6.5 \mathrm{~Hz}, \mathrm{H}-2^{\mathrm{II}}\right)$. Selected ${ }^{13} \mathrm{C}$ NMR $\left(150 \mathrm{MHz}, \mathrm{D}_{2} \mathrm{O}\right): \delta 102.0\left(\mathrm{C}-1^{\mathrm{II}}\right), 89.2\left(\mathrm{C}-2^{\mathrm{I}}\right), 85.7\left(\mathrm{C}-5^{\mathrm{I}}\right), 84.6\left(\mathrm{C}-4^{\mathrm{I}}\right), 82.0\left(\mathrm{C}-2^{\mathrm{I}}\right)$, $76.5\left(\mathrm{C}-3^{\mathrm{I}}\right), 75.1\left(\mathrm{C}-4^{\mathrm{II}}\right), 74.8\left(\mathrm{C}-5^{\mathrm{II}}\right), 72.8\left(\mathrm{C}-2^{\mathrm{II}}\right), 71.6\left(\mathrm{C}-3^{\mathrm{II}}\right)$; HRMS-FAB: $[\mathrm{M}-\mathrm{H}]^{-} \mathrm{m} / z$ calcd for $\mathrm{C}_{12} \mathrm{H}_{20} \mathrm{O}_{12}$ : 355.0877 ; found: 355.0882 .

\section{3. $\beta$-D-glucopyranosiduronate-( $1 \rightarrow 4)-2,5$-Anhydro-D-mannose oxime (6)}

To a solution of $4(450 \mathrm{mg}, 1.33 \mathrm{mmol})$ in water hydroxylamine hydrochloride $(0.11 \mathrm{~g}, 1.59$ $\mathrm{mmol})$ and sodium acetate $(0.16 \mathrm{~g}, 1.91 \mathrm{mmol})$ were successively added. The solution was stirred overnight at room temperature. The solvent was removed under reduced pressure and the resulting syrup was purified by a Biogel P-2 column $(1.5 \times 200 \mathrm{~cm})$ at a flow rate of 15 $\mathrm{ml} / \mathrm{h}$ to give the crude product $6(420 \mathrm{mg}, 89.5 \%)$ as off-white powder $(\mathrm{BuOH} / \mathrm{HCOOH} /$ $\left.\mathrm{H}_{2} \mathrm{O}=4: 8: 1, \mathrm{R}_{\mathrm{f}}=0.51\right) .{ }^{1} \mathrm{H}$ NMR $\left(600 \mathrm{MHz}, \mathrm{D}_{2} \mathrm{O}\right): \delta 7.44(\mathrm{~d}, 1 \mathrm{H}, J=3.78 \mathrm{~Hz}, \mathrm{C} H=\mathrm{N}(E))$, $6.84(\mathrm{~d}, 1 \mathrm{H}, J=6.88 \mathrm{~Hz}, \mathrm{C} H=\mathrm{N}(Z)), 4.38\left(\mathrm{~d}, 1 \mathrm{H}, J=7.8 \mathrm{~Hz}, \mathrm{H}-1^{\mathrm{II}}\right), 4.28\left(\mathrm{~m}, 1 \mathrm{H}, \mathrm{H}-3^{\mathrm{I}}\right)$, 4.05-4.04 (m, $\left.2 \mathrm{H}, \mathrm{H}-4^{\mathrm{I}}, \mathrm{H}^{\mathrm{I}}\right), 3.96\left(\mathrm{~m}, 1 \mathrm{H}, \mathrm{H}-2^{\mathrm{I}}\right), 3.61-3.64\left(\mathrm{~m}, 2 \mathrm{H}, \mathrm{H}-5^{\mathrm{II}}, \mathrm{H}-6 \mathrm{~b}^{\mathrm{I}}\right), 3.57$ $\left.\left(\mathrm{m}, 2 \mathrm{H}, \mathrm{H}-6 \mathrm{a}^{\mathrm{I}}\right)\right), 3.37\left(\mathrm{~m}, 1 \mathrm{H}, \mathrm{H}-3^{\mathrm{II}}, \mathrm{H}-4^{\mathrm{II}}\right), 3.20\left(\mathrm{t}, 1 \mathrm{H}, J=6.5 \mathrm{~Hz}, \mathrm{H}-2^{\mathrm{II}}\right)$. Selected ${ }^{13} \mathrm{C}$ NMR $\left(150 \mathrm{MHz}, \mathrm{D}_{2} \mathrm{O}\right): \delta 102.0\left(\mathrm{C}-1^{\mathrm{II}}\right), 85.1\left(\mathrm{C}^{\mathrm{II}}\right), 82.1\left(\mathrm{C}-3^{\mathrm{I}}\right), 79.4\left(\mathrm{C}-4^{\mathrm{I}}\right), 77.8\left(\mathrm{C}-2^{\mathrm{I}}\right)$,

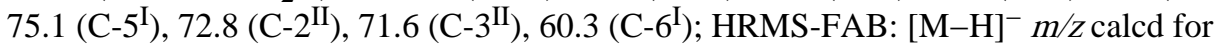
$\mathrm{C}_{12} \mathrm{H}_{18} \mathrm{NO}_{11} 352.0880$; found, 352.0883 .

\section{4. $\beta$-D-glucopyranosiduronate-(1 $\rightarrow$ 4)-1-amino-2,5-anhydro-1-deoxy-D-mannitol (7)}

$\mathrm{Pd}(\mathrm{OH})_{2}-\mathrm{C} 10 \%(0.19 \mathrm{~g})$ was added to a solution of oxime $6(240 \mathrm{mg}, 0.68 \mathrm{mmol})$ in water and several drops of acetic acid were subsequently added. The suspension was stirred under $\mathrm{H}_{2}$ at atmospheric pressure and room temperature for $2 \mathrm{~h}$. The solvent was removed under reduced pressure and the resulting syrup was purified by a Biogel P-2 column $(1.5 \times 200$ $\mathrm{cm})$ at a flow rate of $15 \mathrm{ml} / \mathrm{h}$ to obtain compound $7(210 \mathrm{mg}, 0.62 \mathrm{mmol}, 91 \%)$ as white powder $\left(\mathrm{BuOH} / \mathrm{HCOOH} / \mathrm{H}_{2} \mathrm{O}=4: 8: 1, \mathrm{R}_{\mathrm{f}}=0.28\right) .{ }^{1} \mathrm{H}$ NMR $\left(600 \mathrm{MHz}, \mathrm{D}_{2} \mathrm{O}\right): \delta 4.39(\mathrm{~d}, 1$ $\left.\mathrm{H}, J=7.6 \mathrm{~Hz}, \mathrm{H}-1^{\mathrm{II}}\right), 4.04-4.08(\mathrm{~m}, 2 \mathrm{H}), 3.95(\mathrm{~s}, 1 \mathrm{H}), 3.91(\mathrm{~s}, 1 \mathrm{H}), 3.77-3.84(\mathrm{~m}, 2 \mathrm{H})$, 3.70-3.75 (m, $1 \mathrm{H}), 3.60-3.64$ (m, $2 \mathrm{H}), 3.55-3.59$ (m, 1H), 3.41 (t, $1 \mathrm{H}, J=8.4 \mathrm{~Hz}), 3.36$ $(\mathrm{m}, 1 \mathrm{H})$. Selected ${ }^{13} \mathrm{C}$ NMR $\left(150 \mathrm{MHz}, \mathrm{D}_{2} \mathrm{O}\right): \delta 103.1\left(\mathrm{C}-1^{\mathrm{II}}\right), 80.3,76.6,75.2\left(\mathrm{C}-5^{\mathrm{II}}\right)$, $72.8\left(\mathrm{C}-2^{\mathrm{II}}\right), 71.6\left(\mathrm{C}-3^{\mathrm{II}}\right), 71.0,69.4,68.8,61.9,61.1\left(\mathrm{C}-6^{\mathrm{I}}\right)$; HRMS-FAB: $[\mathrm{M}-\mathrm{H}]^{-} \mathrm{m} / \mathrm{z}$ calcd for $\mathrm{C}_{12} \mathrm{H}_{20} \mathrm{NO}_{10}$ : 338.1087 ; found: 338.1088 .

\section{5. $\beta$-D-glucopyranosiduronate-( $1 \rightarrow 4)-2,5$-anhydro-1-azido-1-deoxy-D-mannitol (1)}

A solution of $\mathrm{NaN}_{3}(992 \mathrm{mg}, 15.2 \mathrm{mmol})$ in $2.5 \mathrm{~mL}$ of $\mathrm{H}_{2} \mathrm{O}$ was cooled to $\sim 0{ }^{\circ} \mathrm{C}$ in an ice bath and subsequently added to $5 \mathrm{~mL}$ of $\mathrm{CH}_{2} \mathrm{Cl}_{2}$. A biphasic mixture generally formed that was stirred vigorously and $\mathrm{Tf}_{2} \mathrm{O}(872 \mathrm{mg}, 3.08 \mathrm{mmol})$ was slowly added drop-wise over a period of $10 \mathrm{~min}$. The reaction was then stirred under ice bath for $2 \mathrm{~h}$, and the organic phase was separated and aqueous phase was extracted with $\mathrm{CH}_{2} \mathrm{Cl}_{2}(2 \times 2.5 \mathrm{~mL})$. Then combined 
organic phase was extracted with saturated $\mathrm{Na}_{2} \mathrm{CO}_{3}$ solution and used without further purification. The final concentration of $\mathrm{TfN}_{3}$ was $0.2 \mathrm{M}$ in $10 \mathrm{~mL}$ solvent.

Substrate 7 (120 mg, $0.35 \mathrm{mmol}$ ) was dissolved in $5 \mathrm{~mL}$ of $\mathrm{MeOH}: \mathrm{H}_{2} \mathrm{O}$ (1: 1). $\mathrm{CuSO}_{4}(2.8$ $\mathrm{mg}, 0.018 \mathrm{mmol})$ and triethylamine $(97 \mu \mathrm{L}, 0.70 \mathrm{mmol}, 2$ equiv per amine substrate) were added to the solution of substrate 7 with stirring. The mixture was cooled in an ice bath for $15 \mathrm{~min}$, and $0.2 \mathrm{M} \mathrm{CH}_{2} \mathrm{Cl}_{2}$ solution of triflyl azide $(2.1 \mathrm{~mL}, 1.2$ equiv per amino group based on the amount of triflic anhydride used in the preparation of $\mathrm{TfN}_{3}$ ) was added into the above reaction solution dropwise. The reaction mixture was allowed to warm to room temperature and a homogeneous solution was obtained after the addition. The reaction was finished in $6 \mathrm{~h}$ as determined by monitoring triflyl azide by TLC $\left(\mathrm{BuOH} / \mathrm{HCOOH} / \mathrm{H}_{2} \mathrm{O}=\right.$ $\left.4: 8: 1, R_{\mathrm{f}}=0.56\right)$. The solvent was removed under reduced pressure and the resulting syrup was purified by chromatography on a Biogel P-2 column $(1.5 \times 200 \mathrm{~cm})$ eluted with water at a flow rate of $15 \mathrm{ml} / \mathrm{h}$. The fractions of the crude product $6(412 \mathrm{mg}, 88 \%)$ as off-white powder were then collected and subjected to NMR and ESI-MS analysis. ${ }^{1} \mathrm{H}$ NMR (600 $\left.\mathrm{MHz}, \mathrm{D}_{2} \mathrm{O}\right): \delta 4.38\left(\mathrm{~d}, 1 \mathrm{H}, J=7.8 \mathrm{~Hz}, \mathrm{H}_{-1}{ }^{\mathrm{II}}\right), 4.13\left(\mathrm{t}, 1 \mathrm{H}, J=5.7 \mathrm{~Hz}, \mathrm{H}-3^{\mathrm{I}}\right), 3.99(\mathrm{~m}, 2 \mathrm{H}$, H-5 $\left.{ }^{\mathrm{I}}, \mathrm{H}-4^{\mathrm{I}}\right), 3.91\left(\mathrm{~s}, 1 \mathrm{H}, \mathrm{H}-2^{\mathrm{I}}\right), 3.64\left(\mathrm{~d}, 1 \mathrm{H}, J=12.0 \mathrm{~Hz}, \mathrm{H}-6 \mathrm{~b}^{\mathrm{I}}\right), 3.63\left(\mathrm{~s}, 1 \mathrm{H}, \mathrm{H}-5^{\mathrm{II}}\right), 3.59$ $\left(\mathrm{dd}, 1 \mathrm{H}, J=4.7,12.4 \mathrm{~Hz}, \mathrm{H}-6 \mathrm{a}^{\mathrm{I}}\right), 3.52\left(\mathrm{~d}, 1 \mathrm{H}, J=13.0 \mathrm{~Hz}, \mathrm{H}-1 \mathrm{~b}^{\mathrm{I}}\right), 3.40-3.35(\mathrm{~m}, 3 \mathrm{H}$, $\left.\mathrm{H}-1 \mathrm{a}^{\mathrm{I}}, \mathrm{H}-4^{\mathrm{II}}, \mathrm{H}-3^{\mathrm{II}}\right), 3.22\left(\mathrm{t}, 1 \mathrm{H}, J=7.92 \mathrm{~Hz}, \mathrm{H}-2^{\mathrm{II}}\right) .{ }^{13} \mathrm{C}$ NMR $\left(150 \mathrm{MHz}, \mathrm{D}_{2} \mathrm{O}\right): \delta 175.6$ $\left(\mathrm{C}-6^{\mathrm{II}}\right), 101.9\left(\mathrm{C}^{\mathrm{II}}\right), 85.4\left(\mathrm{C}-5^{\mathrm{I}}\right), 81.5\left(\mathrm{C}-4^{\mathrm{I}}\right), 80.9\left(\mathrm{C}-2^{\mathrm{I}}\right), 76.5\left(\mathrm{C}^{\mathrm{I}} 3^{\mathrm{I}}\right), 75.3\left(\mathrm{C}-4^{\mathrm{II}}\right), 74.8$ $\left(\mathrm{C}-5^{\mathrm{II}}\right), 72.8\left(\mathrm{C}-2^{\mathrm{II}}\right), 71.5\left(\mathrm{C}-3^{\mathrm{II}}\right), 60.9\left(\mathrm{C}-6^{\mathrm{I}}\right), 51.4\left(\mathrm{C}-1^{\mathrm{I}}\right)$; HRMS-FAB: $[\mathrm{M}-\mathrm{H}]^{-} \mathrm{m} / \mathrm{z}$ calcd for $\mathrm{C}_{12} \mathrm{H}_{18} \mathrm{~N}_{3} \mathrm{O}_{10}, 364.0992$; found: 364.1000 .

\subsection{Methyl 2,3,4-tri-O-acetyl- $\beta$-D-glucopyranosiduronate-(1 $\rightarrow 4)-3,6$-di-0-acetyl-2,5- anhydro-1-azido-1-deoxy-D-mannitol (8)}

The crude compound 1 ( $45 \mathrm{mg}, 0.12 \mathrm{mmol}$ ) was dissolved in $3 \mathrm{~mL}$ methanol, and $90 \mathrm{mg}$ Amberlite $120\left(\mathrm{H}^{+}\right)$resin was added into the solution. The mixture was vigorously stirred for $12 \mathrm{~h}$ at room temperature. The resin was filtered to obtain the organic solvent, which was concentrated under vacuum. The resulting residue was dissolved in $2 \mathrm{~mL}$ pyridine and $1 \mathrm{~mL}$ $\mathrm{Ac}_{2} \mathrm{O}$ and was stirred for $12 \mathrm{~h}$ at room temperature. The organic solvent was concentrated under reduced pressure, and the residue was purified by flash silica column chromatography (EtOAc-Hexanes 1:2) giving compound $8(67 \mathrm{mg}, 95 \%)$ as syrupy. ${ }^{1} \mathrm{H}$ NMR (600 MHz, $\left.\mathrm{CDCl}_{3}\right): \delta 5.19\left(\mathrm{t}, 1 \mathrm{H}, J=9.5 \mathrm{~Hz}, \mathrm{H}-4^{\mathrm{II}}\right), 5.16\left(\mathrm{~d}, 1 \mathrm{H}, J=9.0 \mathrm{~Hz}, \mathrm{H}-3^{\mathrm{II}}\right), 5.14(\mathrm{t}, 1 \mathrm{H}, J=$ $\left.3.5 \mathrm{~Hz}, \mathrm{H}-3^{\mathrm{I}}\right), 4.95\left(\mathrm{t}, 1 \mathrm{H}, J=8.5 \mathrm{~Hz}, \mathrm{H}-2^{\mathrm{II}}\right), 4.63\left(\mathrm{~d}, 1 \mathrm{H}, J=7.5 \mathrm{~Hz}, \mathrm{H}-1^{\mathrm{II}}\right), 4.14-4.16(\mathrm{~m}$, $\left.1 \mathrm{H}, \mathrm{H}-4^{\mathrm{I}}\right), 4.13\left(\mathrm{t}, 1 \mathrm{H}, J=3.5 \mathrm{~Hz}, \mathrm{H}-2^{\mathrm{I}}\right), 4.09-4.11\left(\mathrm{~m}, 1 \mathrm{H}, \mathrm{H}-6 \mathrm{~b}^{\mathrm{I}}\right), 4.03-4.09(\mathrm{~m}, 2 \mathrm{H}$, $\left.\mathrm{H}-5^{\mathrm{I}}, \mathrm{H}-6 \mathrm{a}^{\mathrm{I}}\right), 3.99\left(\mathrm{~d}, 1 \mathrm{H}, J=9.5 \mathrm{~Hz}, \mathrm{H}-5^{\mathrm{II}}\right), 3.68(\mathrm{~s}, 3 \mathrm{H}, \mathrm{OMe}), 3.46$ (dd, $1 \mathrm{H}, J=7.5,13.0$ $\mathrm{Hz}, \mathrm{H}-1 \mathrm{~b}^{\mathrm{I}}$ ), 3.39 (dd, $\left.1 \mathrm{H}, J=4.5,13.0 \mathrm{~Hz}, \mathrm{H}-1 \mathrm{a}^{\mathrm{I}}\right), 2.05$ (s, $\left.3 \mathrm{H}, A c\right), 2.01$ (s, 3H, $\left.A c\right), 1.99$ $(\mathrm{s}, 3 \mathrm{H}, A c), 1.96(\mathrm{~s}, 2 \times 3 \mathrm{H}, A c) .{ }^{13} \mathrm{C}$ NMR $\left(150 \mathrm{MHz}, \mathrm{CDCl}_{3}\right): \delta 170.5\left(\mathrm{C}-6^{\mathrm{II}}\right), 166.9$ $(A c), 100.6\left(\mathrm{C}-1^{\mathrm{II}}\right), 84.6\left(\mathrm{C}-5^{\mathrm{I}}\right), 82.8\left(\mathrm{C}-2^{\mathrm{I}}\right), 80.2\left(\mathrm{C}-4^{\mathrm{I}}\right), 79.3\left(\mathrm{C}-3^{\mathrm{I}}\right), 72.6\left(\mathrm{C}-5^{\mathrm{II}}\right), 71.8$ $\left(\mathrm{C}-4^{\mathrm{II}}\right), 70.8\left(\mathrm{C}-2^{\mathrm{II}}\right), 69.1\left(\mathrm{C}-3^{\mathrm{II}}\right), 63.0\left(\mathrm{C}-6^{\mathrm{I}}\right), 53.0(\mathrm{OMe}), 51.9\left(\mathrm{C}-1^{\mathrm{I}}\right)$; HRMS-FAB: [M $+\mathrm{H}]^{+} \mathrm{m} / \mathrm{z}$ calcd for $\mathrm{C}_{23} \mathrm{H}_{32} \mathrm{~N}_{3} \mathrm{O}_{15}, 590.1833$; found, 590.1840.

\section{7. $\beta$-D-glucopyranosiduronate-(1 $\rightarrow 4)$ - $\alpha$-D-2-trifluroacetamido-2-deoxy-glucopyranosyl- $(1 \rightarrow 4)$ - $\beta$-D-glucopyranosiduronate-( $\rightarrow 4)$-2,5-anhydro-1-azido-1-deoxy-D-mannitol (9)}

To synthesize oligosaccharide backbone, disaccharide (GlcA-AnMan- $\left.\mathrm{N}_{3}, \mathbf{1}\right)(3.6 \mathrm{mg}, 10$ $\mu \mathrm{mol})$ was incubated with UDP-GlcNTFA $(8.5 \mathrm{mg}, 12 \mu \mathrm{mol})$ and $\mathrm{KfiA}(0.1 \mathrm{mg})$ in $10 \mathrm{ml}$ of buffer containing $25 \mathrm{mM}$ Tris- $\mathrm{HCl}(\mathrm{pH} 7.2)$ and $10 \mathrm{mM} \mathrm{MgCl}_{2}$. The reaction was incubated at room temperature overnight, and the reaction mixture was analyzed by a polyamine-based HPLC column to ensure that 95\% of UDP-GlcNTFA was converted to UDP. Then $10-\mu \mathrm{L}$ of reaction solution was passed through a $3000 \mathrm{MWCO}$ spin-column and subjected to mass spectral analysis. HRMS-FAB: $[\mathrm{M}-\mathrm{H}]^{-} \mathrm{m} / \mathrm{z}$ calcd for $\mathrm{C}_{20} \mathrm{H}_{28} \mathrm{~F}_{3} \mathrm{~N}_{4} \mathrm{O}_{15}, 621.1503$; found: 621.1509. Upon the complete consumption of UDP-GlcNTFA, pmHS2 $(0.1 \mathrm{mg})$ and UDP- 
GlcA ( $7.5 \mathrm{mg}, 12 \mu \mathrm{mol}$ ) were added into the reaction mixture for additional $4-5 \mathrm{~h}$ at room temperature. Another portion of pmHS2 $(0.1 \mathrm{mg})$ and UDP-GlcA $(12 \mu \mathrm{mol})$ was added to drive the transfer of GlcUA unit to completion. The product was purified using Biogel P-2 chromatography on a column $(1.5 \times 200 \mathrm{~cm})$ that was equilibrated with $0.1 \mathrm{M}$ ammonium bicarbonate at a flow rate of $15 \mathrm{ml} / \mathrm{h}$. The fraction containing the product was lyophilized to give $9(6.4 \mathrm{mg}, 80 \%)$ and subjected to NMR and ESI-MS analysis. ${ }^{1} \mathrm{H}$ NMR $(600 \mathrm{MHz}$, $\left.\mathrm{D}_{2} \mathrm{O}\right): \delta 5.25-5.31(\mathrm{~m}, 1 \mathrm{H}), 4.38(\mathrm{~d}, 1 \mathrm{H}, J=9.6 \mathrm{~Hz}), 4.35(\mathrm{~d}, 1 \mathrm{H}, J=9.6 \mathrm{~Hz}), 4.29(\mathrm{~s}, 1$ H), $4.14(\mathrm{t}, 1 \mathrm{H}, J=5.4 \mathrm{~Hz}), 4.03(\mathrm{t}, 1 \mathrm{H}, J=5.4 \mathrm{~Hz}), 3.98$ (dd, $1 \mathrm{H}, J=5.4,10.2 \mathrm{~Hz}), 3.93-$ $3.95(\mathrm{~m}, 1 \mathrm{H}), 3.90(\mathrm{dd}, 1 \mathrm{H}, J=5.4,10.2 \mathrm{~Hz}), 3.84-3.87(\mathrm{~m}, 1 \mathrm{H}), 3.69-3.75(\mathrm{~m}, 2 \mathrm{H})$, 3.57-3.69 (m, $4 \mathrm{H}), 3.52-3.56(\mathrm{~m}, 1 \mathrm{H}), 3.44-3.50(\mathrm{~m}, 2 \mathrm{H}), 3.34-3.39(\mathrm{~m}, 3 \mathrm{H}), 3.22(\mathrm{t}, 2$ $\mathrm{H}, J=8.2 \mathrm{~Hz}$ ). HRMS-FAB: [M-H] $]^{-} \mathrm{m} / \mathrm{z}$ calcd for $\mathrm{C}_{26} \mathrm{H}_{36} \mathrm{~F}_{3} \mathrm{~N}_{4} \mathrm{O}_{21}$, 797.1824; found: 797.1832 .

\section{Supplementary Material}

Refer to Web version on PubMed Central for supplementary material.

\section{Acknowledgments}

This work was supported by the National Institutes of Health in the form of grant \# HL62244, GM38060 and HL094463.

\section{References}

1. Linhardt RJ. J Med Chem. 2003; 46:2551-2554. [PubMed: 12801218]

2. Esko JD, Lindahl U. J Clin Invest. 2001; 108:169-173. [PubMed: 11457867]

3. Gandhi NS, Mancera RL. Chem Biol Drug Des. 2008; 72:455-482. [PubMed: 19090915]

4. Parish CR. Nat Rev Immunol. 2006; 6:633-643. [PubMed: 16917509]

5. Sasisekharan R, Shriver Z, Venkataraman G, Narayanasami U. Nat Rev Cancer. 2002; 2:521-528. [PubMed: 12094238]

6. Sinaÿ P, Jacquinet JC, Petitou M, Duchaussoy P, Lederman I, Choay J, Torri G. Carbohydr Res. 1984; 132:C5-C9.

7. Cai C, Solakyildirim K, Yang B, Beaudet JM, Weyers A, Linhardt RJ, Zhang F. Carbohydr Polym. 2012; 87:822-829. [PubMed: 22140285]

8. Noti, C.; Seeberger, PH. Chemistry and Biology of Heparin and Heparan Sulfate. Garg, HG.; Linhardt, RJ.; Hales, CA., editors. Elsevier; Oxford: 2005. p. 79-142.

9. Arungundram S, Boons GJ. J Am Chem Soc. 2009; 131:17394-17405. [PubMed: 19904943]

10. Wang Z, Xu Y, Yang B, Tiruchinapally G, Sun B, Liu R, Dulaney S, Liu J, Huang X. Chem Eur J. 2010; 16:8365-8375. [PubMed: 20623566]

11. Hu Y-P, Lin S-Y, Huang C-Y, Zulueta MML, Liu J-Y, Chang W, Huang S-C. Nat Chem. 2011; 3:557-563. [PubMed: 21697878]

12. Linhardt RJ, Liu J. Current Opinion in Pharmacology. 2012; 12:217-219. [PubMed: 22325855]

13. Liu R, Xu Y, Chen M, Weïwer M, Zhou X, Bridges AS, DeAngelis PL, Zhang Q, Linhardt RJ, Liu J. J Biol Chem. 2010; 285:34240-34249. [PubMed: 20729556]

14. Xu Y, Masuko S, Takieddin M, Xu H, Liu R, Jing J, Mousa S, Linhardt RJ, Liu J. Science. 2011; 334:498-501. [PubMed: 22034431]

15. Masuko S, Linhardt RJ. Future Med Chem. 2012; 4:289-296. [PubMed: 22393937]

16. Chen M, Bridges A, Liu J. Biochemistry. 2006; 45:12358-12365. [PubMed: 17014088]

17. Kane TA, White CL, DeAngelis PL. J Biol Chem. 2006; 281:33192-33197. [PubMed: 16959770]

18. Kuberan B, Lech MZ, Beeler DL, Wu ZL, Rosenberg RD. Nat Biotechnol. 2003; 21:1343-1346. [PubMed: 14528313]

19. Shaklee PN, Conrad HE. Biochem J. 1986; 235:225-236. [PubMed: 3741382] 
20. Wang Z, Yang B, Zhang Z, Ly M, Takieddin M, Mousa S, Liu J, Dordick JS, Linhardt RJ. Appl Microbiol Biotechnol. 2011; 91:91-99. [PubMed: 21484210]

21. Kariya Y, Herrmann J, Suzuki K, Isomura T, Ishihara M. J Biochem. 1998; 123:240-246. [PubMed: 9538198]

22. Chen S-Y, Joullié. J Org Chem. 1984; 49:1769-1772.

23. Blundell CD, Reed Michelle AC, Almond A. Carbohydr Res. 2006; 341:2803-2815. [PubMed: 17056022]

24. Abdel-Rahman A-H, E1 Ashry EH, Schmidt RR. Carbohydr Res. 1999; 315:106-116.

25. Nyffeler PT, Liang C-H, Koeller KM, Wong C-H. J Am Chem Soc. 2002; 124:10773-10778. [PubMed: 12207533] 


\section{Highlights}

- A disaccharide was prepared by chemical treatment of heparosan

- An azido group was introduced into this heparosan disaccharide

- The azido heparosan disaccharide was used as a glycosylation acceptor

- A heparin tetrasaccharide was enzymatically synthesized 


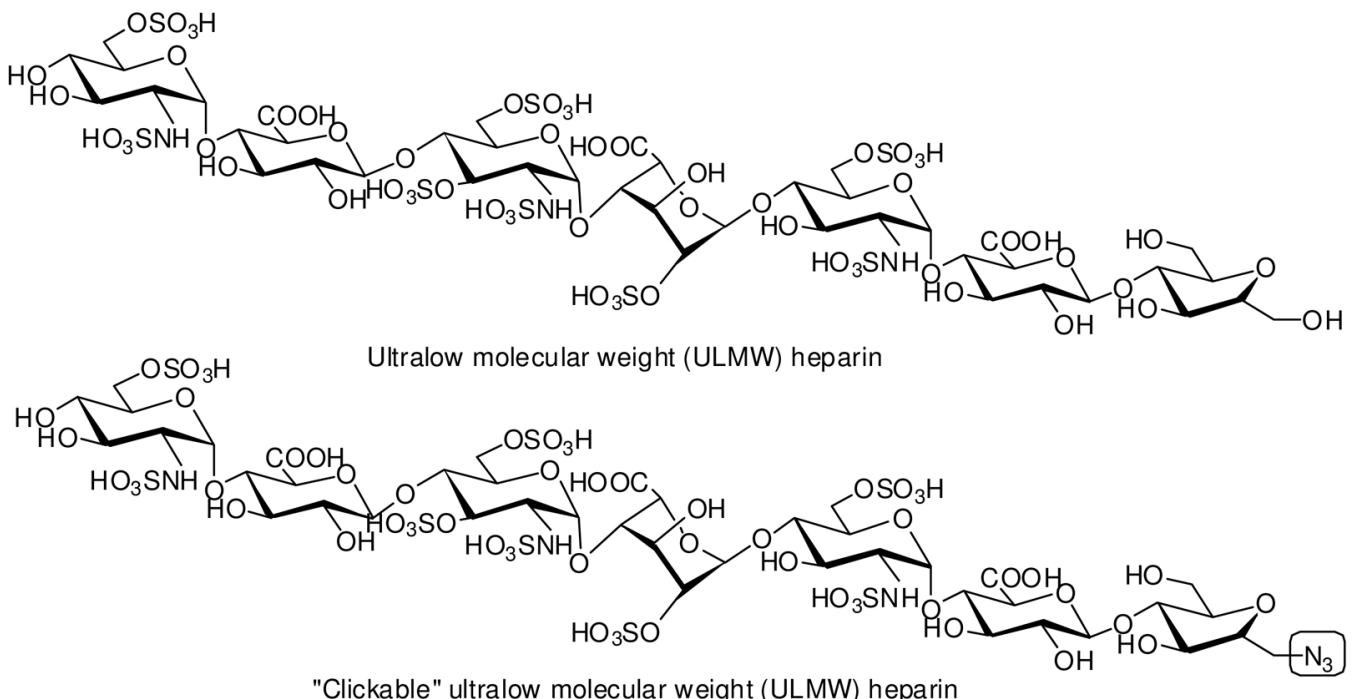

Figure 1.

Structures of targeted ULMW heparin oligosaccharides 

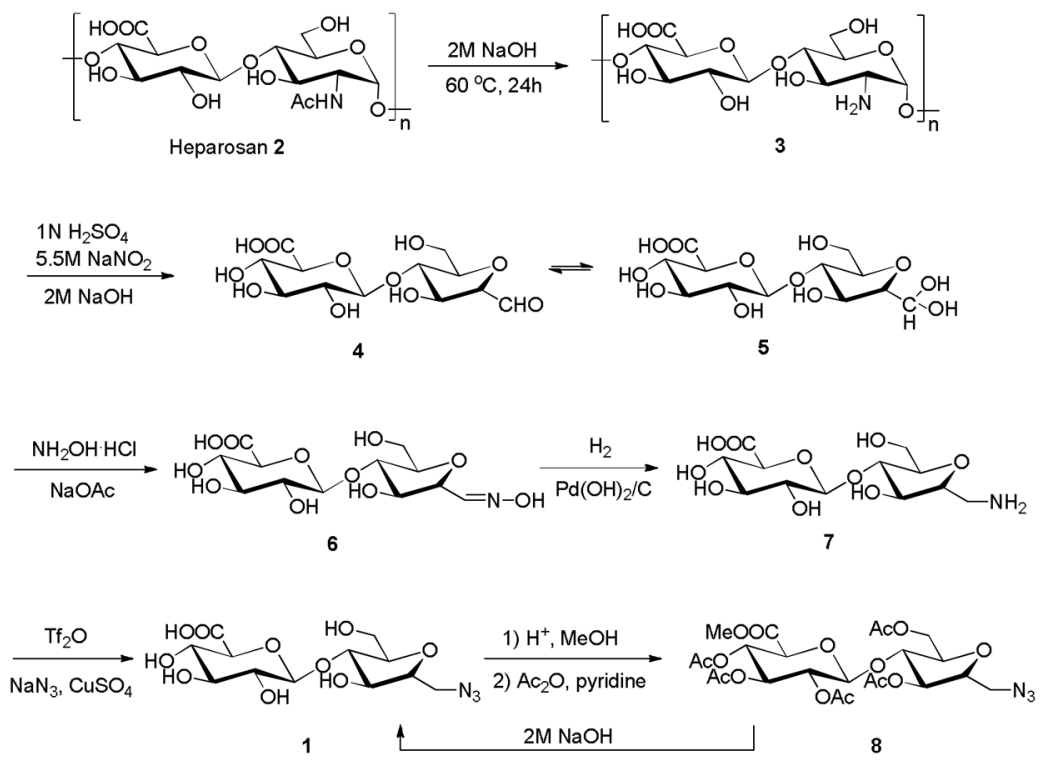

Scheme 1.

Synthesis of "clickable" disaccharide acceptor $\mathbf{1}$ 


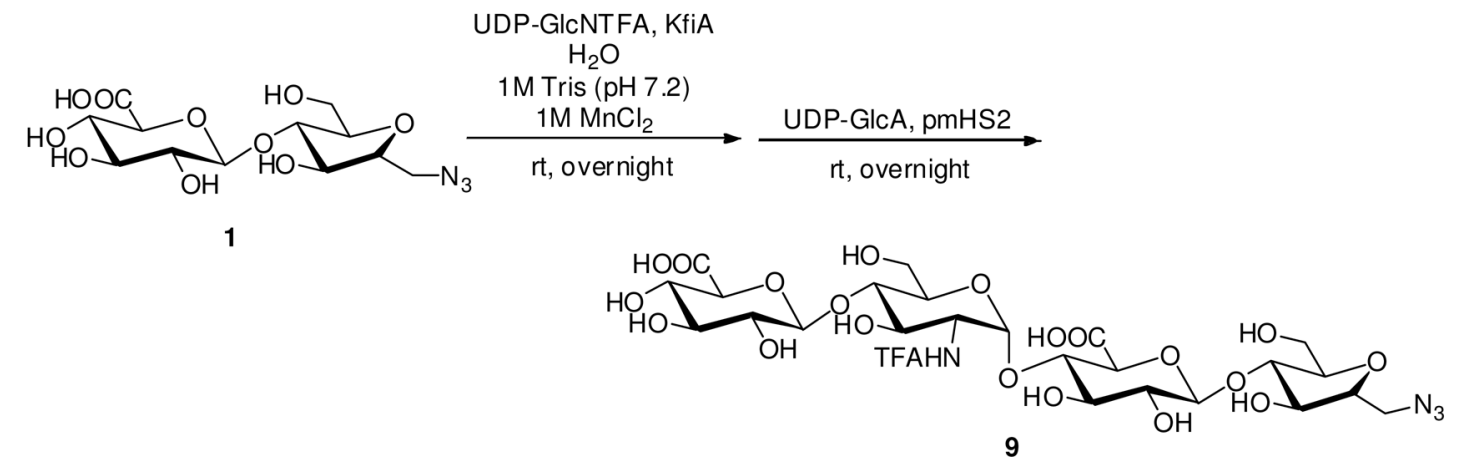

Scheme 2.

Chemoenzymatic synthesis of HS tetrasaccharide 